\title{
Fiber-coupled erbium microlasers on a chip
}

\author{
Lan Yang, ${ }^{\text {a) }}$ D. K. Armani, and K. J. Vahala ${ }^{\text {b) }}$ \\ Department of Applied Physics, California Institute of Technology, Pasadena, California 91125
}

(Received 21 April 2003; accepted 13 June 2003)

\begin{abstract}
An erbium-doped, toroid-shaped microlaser fabricated on a silicon chip is described and characterized. Erbium-doped sol-gel films are applied to the surface of a silica toroidal microresonator to create the microcavity lasers. Highly confined whispering gallery modes make possible single-mode and ultralow threshold microlasers. (c) 2003 American Institute of Physics. [DOI: $10.1063 / 1.1598623]$
\end{abstract}

Whispering-gallery type microlasers in which the cavity boundary is defined by surface tension (e.g., spheres and droplets) have attracted much attention because the combination of their very small mode volume and high, cold-cavity $Q$ factor enables ultralow threshold operation. ${ }^{1-3}$ Recently, a class of ultrahigh- $Q$, surface-tension-induced microcavities fabricated on a silicon chip have been demonstrated. ${ }^{4}$ These structures feature a toroidal-shaped cavity and enable the integration of electronics and other functions with ultrahigh- $Q$ devices. In this letter we demonstrate surface functionalization of these devices using erbium-doped sol-gel films. In addition to being integrable with other optical or electric components, they are directly coupled to optical fiber using fiber tapers.

We have previously applied the surface functionalization method using silica microsphere resonators. ${ }^{5}$ Erbium-doped microlasers are especially interesting because their emission band falls in the important $1.5 \mu \mathrm{m}$ window used for optical communications. However, microspheres, while useful as laboratory demonstration vehicles, are not suitable for integration with other optical or electronic functions. Their properties are also difficult to control during fabrication. In contrast, microlasers on a chip can be fabricated in parallel and have characteristics that are more easily controlled using wafer-scale processing methods.

Silica toroid-shaped microresonators supported by a circular silicon pillar were fabricated upon a silicon wafer containing a $2 \mu \mathrm{m}$ layer of thermal silica $\left(\mathrm{SiO}_{2}\right) .{ }^{4}$ The process details are described in Ref. 4. The sol-gel starting solution was prepared as described in Ref. 5. After aging the sol-gel solution at room temperature for $10 \mathrm{~h}$, we immersed silica microtoroids in the solution for 3-5 h. Then the wafers were heated in an oven at $160^{\circ} \mathrm{C}$ for another $10 \mathrm{~h}$ to drive off surface water. Microtoroids were then irradiated with a $\mathrm{CO}_{2}$ laser (10.6 $\mu \mathrm{m}$ emission) in order to reflow and densify the sol-gel films. As described in Ref. 4, $\mathrm{CO}_{2}$-laser emission is selectively absorbed by the silica layers. This and the relatively high thermal conductivity of silicon $(\sim 100$ times more thermally conductive than silica) ${ }^{6,7}$ lead to selective reflow and densification of sol-gel at the all-important toroid periphery. Sol-gel deposited elsewhere was unaffected by this process step. Because of the large difference between the

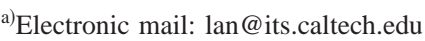

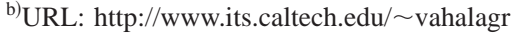

etching rate of densified and undensified silica films in buffered $\mathrm{HF}^{8}{ }^{8}$ sol-gel deposited on all regions of the wafer other than the densified perimeter of the toroid could subsequently be selectively removed. Microtoroids ranging in diameter from 60 to $85 \mu \mathrm{m}$ were fabricated and the $\mathrm{Er}^{3+}$ concentration in the sol-gel layer was estimated to be around $10^{19} \mathrm{~cm}^{-3}$.

Like all whispering-gallery type microresonators, microtoroids feature optical modes that are confined near the resonator periphery. The thickness of the microtoroid is generally much smaller than the microtoroid diameter. Thus, in contrast to microspheres, these structures support fewer azimuthal modes. This facilitates single-mode operation in the microtoroids. An important feature of gain functionalization of the surface is that it puts optical gain only where it is needed, i.e., where the best overlap is possible with the fundamental whispering gallery modes.

Figure 1 illustrates both a sol-gel functionalized microtoroid and the taper coupling configuration used to both provide optical pumping and to extract laser optical power. The inset of Fig. 1 is a photomicrograph showing a taper-coupled microtoroid laser. The pump wave was provided by a tunable, single-frequency, external-cavity laser operating in the $980 \mathrm{~nm}$ band and having a short-term linewidth less than 300 $\mathrm{kHz}$. The sample chip was held in a rotator that was mounted

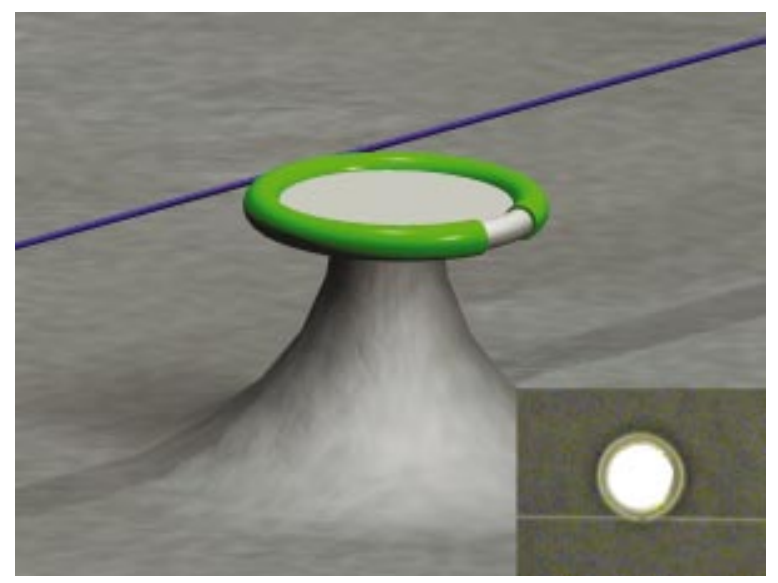

FIG. 1. (Color) Illustration showing a microchip laser consisting of an erbium-doped sol-gel thin film applied to a microtoroid. Also shown is a fiber taper used for both pump coupling and laser emission extraction. The inset is a photomicrograph of the top view of an erbium microtoroid laser coupled by a fiber taper. 


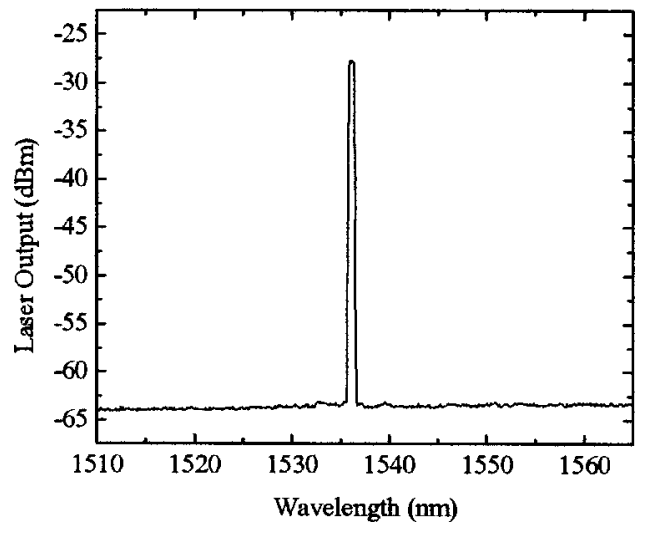

FIG. 2. Emission spectrum of a microtoroid laser with approximate diameter of $80 \mu \mathrm{m}$.

upon a three-axis translator for position control. Two cameras were used to monitor the microtoroid samples and the taper, providing both horizontal and vertical views. The angle of the microtoroid relative to the taper was adjusted using the rotator to align the taper with the equatorial plane of the toroid.

An optical spectrum analyzer (OSA) with resolution of $0.5 \mathrm{~nm}$ was used to measure the laser emission. A typical laser spectrum is presented in Fig. 2. Single line emission (within the resolution of the OSA) was most often observed, however, at increased pumping levels it was sometimes possible to induce oscillation in other longitudinal modes. To further resolve the single line observed in the OSA scan of Fig. 2, a high finesse $(\sim 5000)$ Fabry-Perot etalon having a resolution of a few megahertz was also used to analyze the laser spectrum. A single-frequency, tunable, external-cavity laser emitting in the $1500 \mathrm{~nm}$ band and with known short-

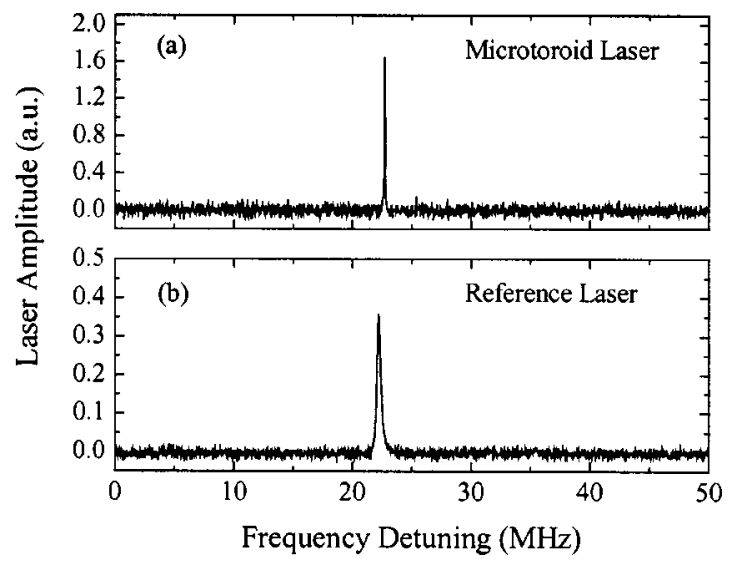

FIG. 3. (a) Laser emission spectrum from Er-doped sol-gel thin-film coated microtoroid laser. (b) Reference laser emission spectrum from a $1550 \mathrm{~nm}$ single-mode laser with short-term linewidth of $300 \mathrm{kHz}$. Both spectra were measured using a high finesse scanning Fabry-Perot spectrometer.

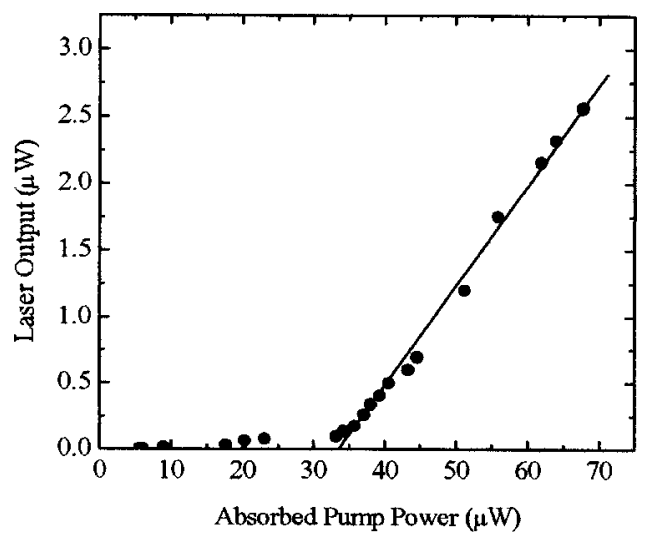

FIG. 4. Measured laser output power plotted vs absorbed pump power for a microtoroid laser with a diameter of $80 \mu \mathrm{m}$.

term linewidth of $300 \mathrm{kHz}$ was measured as a reference. Both spectra are presented in Fig. 3.

The measured laser output power plotted versus the absorbed pump power is shown in Fig. 4. The threshold pump power in this data is $34 \mu \mathrm{W}$ by extrapolation of the linear lasing region. The differential quantum efficiency was measured to be as high as $11 \%$ for the single-mode, unidirectional operation. During measurements, the microtoroids were in contact with the taper. While this creates a very stable coupling, it prevents optimization of the pump and emission coupling efficiencies. As a result, it might be possible to further reduce the threshold in more optimally coupled structures. The effect of optimization of coupling will be part of a future study employing an improved experimental setup.

In summary, we have demonstrated erbium-doped microtoroid lasers on a chip by use of a sol-gel surfacefunctionalization technique. Single-line laser emission and threshold pump powers as low as $34 \mu \mathrm{W}$ were observed. Future work will be directed towards studies of optimal coupling and potential integration with other devices.

This work was supported by the Defense Advanced Research Project Agency, the National Science Foundation, and the Caltech Lee Center. The authors thank Bumki Min for useful discussion.

${ }^{1}$ M. Cai, O. Painter, and K. J. Vahala, Opt. Lett. 25, 1430 (2000).

${ }^{2}$ V. Sandoghdar, F. Treussart, J. Hare, V. Lefevre-Seguin, J.-M. Raimond, and S. Haroche, Phys. Rev. A 54, R1777 (1996).

${ }^{3}$ S. Chang, N. B. Rex, R. K. Chang, G. Chong, and L. J. Guido, Appl. Phys. Lett. 75, 166 (1999).

${ }^{4}$ D. K. Armani, T. K. Kippenberg, S. M. Spillane, and K. J. Vahala, Nature (London) 421, 925 (2003).

${ }^{5}$ L. Yang and K. J. Vahala, Opt. Lett. 28, 592 (2003).

${ }^{6}$ A. D. McLachlan and F. P. Meyer, Appl. Opt. 26, 1728 (1987).

${ }^{7}$ M. Sheik-Bahae and H. S. Kwok, J. Appl. Phys. 63, 518 (1988).

${ }^{8}$ J. W. Zhai, L. Y. Zhang, and X. Yao, J. Inorgan. Mater. 14, 133 (1999). 